\title{
Comunidade Formativa do Estoril: uma comunidade de prática centrada no internato médico e orientada para o futuro
}

Vanessa Sá, ${ }^{1}$ Magda Coutinho, ${ }^{2}$ Ana Póvoa, ${ }^{3}$ Rita Medeiros, ${ }^{1}$ Joana Santos, ${ }^{3}$ André Biscaia, ${ }^{1}$ Isabel Couto ${ }^{2}$

\begin{abstract}
RESUMO
Introdução: Uma comunidade de prática consiste num grupo de pessoas que se unem em torno de um mesmo tópico ou interesse, trabalhando em conjunto para melhorar o que fazem através de interações regulares. A Comunidade Formativa do Estoril reúne o grupo de orientadores e internos de medicina geral e familiar e saúde pública da zona do Estoril, com o objetivo de alargar o seu leque de experiências e vivências, espelhando os princípios de uma comunidade de prática. Este artigo relata a experiência desta Comunidade e tem como principal objetivo dar a conhecer as suas práticas, incentivar a partilha de experiências entre grupos e inspirar a formação de novas comunidades.

Descrição: A Comunidade Formativa do Estoril nasceu no ano de 2015, composta pelos internos e orientadores de formação específica em medicina geral e familiar e de saúde pública de quatro das unidades funcionais do edifício de São João do Estoril do Agrupamento de Centros de Saúde (ACeS) de Cascais. Desde a criação da Comunidade, têm vindo a ser desenvolvidas várias atividades, como reuniões periódicas, elaboração do jornal e das I Jornadas da Comunidade Formativa do Estoril, entre outras.

Conclusão: A Comunidade Formativa representa uma mais-valia para orientadores e internos, numa interação em que o todo é mais vasto do que a soma das partes. No caso da Comunidade Formativa do Estoril a proximidade originada pelo trabalho no mesmo edifício permitiu aos seus membros gerar este projeto, numa atmosfera colaborativa e de interajuda - uma comunidade frutífera, permeável ao que a rodeia e tendo sempre presente o desejo e a capacidade para evoluir através do contributo de cada novo membro e das interações que daí se criam.
\end{abstract}

Palavras-chave: Educação médica; Comunidade formativa; Internato e residência.

\section{INTRODUÇÃO}

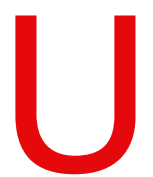

ma comunidade de prática consiste num grupo de pessoas que se unem em torno de um mesmo tópico ou interesse, trabalhando em conjunto para melhorar o que fazem através da interação regular. Uma Comunidade Formativa é uma evolução do conceito de comunidade de prática, sendo uma comunidade multipolar de aprendizagem/formação, que integra várias unidades, re-

1. USF Marginal (ACeS Cascais)

2. USF Costa do Estoril (ACeS Cascais)

3. USF S. João do Estoril (ACeS Cascais) cursos e/ou contextos (diferentes modelos de unidades funcionais, por exemplo). ${ }^{1-5}$

Revendo-se neste conceito, a Comunidade Formati$v a(\mathrm{CF})$ do Estoril reúne habitualmente um grupo de orientadores e internos de São João do Estoril, com o objetivo de alargar o seu leque de experiências e vivências para além da «redoma» muitas vezes criada no local de trabalho, permitindo: ${ }^{1}$

- à equipa interno-orientador: a aquisição e partilha de novos conhecimentos; a geração e discussão de ideias; o desenvolvimento e aperfeiçoamento de projetos, obtendo feedback de outros membros da 
comunidade; o recrutamento de membros para projetos; a dinamização de momentos de partilha abertos a não-membros, como as Jornadas Formativas; a exposição à crítica num contexto propício ao desenvolvimento; o confronto com a realidade de outras listas de utentes e com outros métodos de trabalho; a vigilância e partilha de novidades e inovações na área da formação e da saúde;

- ao interno: o (re)conhecimento de realidades distintas da lista do orientador de formação e da unidade em que se insere; o treino e aprendizagem de aptidões e competências específicas; o fomento do trabalho de equipa com novos colegas; a discussão da adequação dos processos relativos ao internato médico; a partilha de dificuldades; a possibilidade de apoio e partilha na resolução de problemas na relação com o seu orientador e/ou com as estruturas do internato;

- ao orientador: a atualização técnico-científica; a discussão da adequação dos processos relativos à formação e ao internato médico; a discussão de metodologias com outros orientadores de formação; a possibilidade de apoio e partilha na resolução de problemas na relação com o seu interno e/ou com as estruturas do internato.

Este artigo pretende relatar a experiência da CF do Estoril, existente desde 2015. Tem como principal objetivo dar a conhecer as suas práticas, inspirar a formação de novas comunidades e incentivar a partilha de experiências entre comunidades formativas.

\section{A MGF e as Comunidades Formativas}

No início da especialidade, os estágios que constituíam o Internato de Medicina Geral e Familiar (MGF), anteriormente designada por Clínica Geral, eram realizados em meio hospitalar e o estágio em MGF surgiu mais tarde, em 1987, "para que, além de estágios hospitalares, o internato passasse a incluir na sua estrutura um estágio em centro de saúde que mimetizasse o exercício profissional futuro" ${ }^{6-7}$ A relação principal de cada interno era com um dos diretores do internato, tendo o orientador apenas um papel de facilitador do campo do estágio principal. Seguiu-se o primado da relação interno-orientador, ${ }^{8}$ em que esta se assumiu como central na formação, estando o orientador nos vários momentos de avaliação e os diretores do interna- to na supervisão pedagógica e gestão administrativa do internato. No Memorando sobre a formação em MGFe respetivo enquadramento 2007, da Coordenação do Internato Médico de Medicina Geral e Familiar da Zona Sul, dizia-se que "aos orientadores de formação cabe a concretização do Programa do Internato, num processo de orientação direto, ombro a ombro, constituindo a Equipa Integrada de Orientadores a estrutura de apoio e desenvolvimento que permite ultrapassar os constrangimentos do isolamento de exercício". ${ }^{9}$ Mais recentemente surge a ideia de que esta relação interno-orientador deve estar mais diluída numa relação interno-unidade formadora, com os orientadores menos presentes nas avaliações e estas cada vez mais rigidamente estruturadas e entregues a comissões nacionais, regionais ou locais, garantindo uma maior uniformidade.

As Equipas Integradas de Orientadores (EIO) surgem em 2002, na zona sul do país, na sequência da constante evolução da relação dos orientadores e internos com a Coordenação do Internato de MGF, alimentada pelo número crescente de internos e pela necessidade de acompanhar o desenvolvimento das teorias de ensino/aprendizagem.*

O objetivo era estabelecer "percursos formativos, individuais e de grupo (EIO), que resultam do confronto entre as competências exigidas (perfil de competências e necessidades concretas emergentes da prática pedagógica) e as já adquiridas. A EIO, sendo um recurso formativo para o orientador, é também veículo de disseminação entre orientadores das competências adquiridas por cada um, em diversos cenários formativos complementares". ${ }^{9}$

Subjacente a esta evolução está a teoria dos processos proximais do Modelo Bioecológico do Desenvolvimento de Bronfenbrenner que defende que, para ser efetiva, uma interação tem que ocorrer numa base regular e em períodos estendidos no tempo. ${ }^{10}$ Nesta teoria o contexto primordial de desenvolvimento é aquele em que a pessoa pode observar e comprometer-se em atividades conjuntas gradualmente mais complexas $\mathrm{e}$

\footnotetext{
* Os próximos três parágrafos decorreram de uma entrevista a Teresa Ventura, sobre textos dos diretores do Internato de Medicina Geral e Familiar de 2002, da autoria de Francisco Carvalho, Teresa Ventura e Ricardina Barroso.
} 
com a orientação de uma pessoa, ou pessoas, que já possuem habilidade e conhecimentos que ela ainda não possui e com quem ela tenha uma relação afetiva positiva. O contexto secundário de desenvolvimento é aquele em que a pessoa é encorajada a fazer aquilo que desenvolveu no contexto primordial, sendo-lhe dadas as condições e as oportunidades para tal, sem a orientação direta de outras pessoas. Como tal, as EIO foram pensadas como sendo comunidades de aprendizagem recíproca, onde deverão ser criadas oportunidades e condições para que os orientadores possam expressar as suas dificuldades e problemas, apresentar casos reais e demonstrar as soluções que tenham desenvolvido, para além de pesquisarem, experimentarem e partilharem métodos e recursos pedagógicos úteis à orientação pedagógica do internato. Deste modo, há uma apropriação por parte de cada orientador daquilo que de melhor existe na prática dos outros, ocorrendo transferência de saberes entre os elementos da EIO.

Como recurso de formação dos orientadores, estas equipas têm a vantagem de ter um caráter contínuo e de não provocar grandes disrupções no quotidiano de trabalho dos envolvidos. Adicionalmente, as EIO devem contribuir para a melhoria global da qualidade organizacional da direção do internato, criando oportunidades de agilização de processos de comunicação entre a direção e os orientadores e facilitar o envolvimento dos orientadores na definição e na elaboração de instrumentos pedagógicos. Neste sentido, o contributo das EIO é particularmente valioso pelo facto de resultar da discussão e reflexão em grupo.

Posteriormente, surgiram as Equipas Integradas de Internos (EII) com os mesmos objetivos da dos orientadores e, depois, as Equipas Integradas de Orientadores e Internos (EIOI), reunindo orientadores e internos.

\section{DESCRIÇÃO}

No ano de 2015 surgiu a CF do Estoril quando as três unidades de saúde do edifício do ACeS de Cascais, localizado em São João do Estoril, integraram internos e orientadores de MGF num mesmo grupo. Desde então, este grupo tem recebido novos orientadores e internos de MGF e, em 2018, foram também integrados os internos e orientadores de formação específica em Saúde Pública.

Em 2019, a CF do Estoril era constituída por dezassete internos de MGF e três internos de Saúde Pública, quinze orientadores de formação de MGF e dois orientadores de formação de Saúde Pública, pertencentes à Unidade de Saúde Familiar (USF) Costa do Estoril, USF Marginal, USF São João do Estoril e Unidade de Saúde Pública Amélia Leitão. Desde 2007, já se formaram nestas unidades vinte e nove especialistas em MGF e quatro especialistas em Saúde Pública.

A CF baseia-se em cinco princípios: ${ }^{1}$

\section{Modelo centrado nos internos e na aprendizagem}

Esta CF centra-se nos processos de aprendizagem e treino profissional dos internos. Subsidiariamente, também os formadores beneficiam, no seu desenvolvimento profissional e pessoal, das dinâmicas desenvolvidas na $\mathrm{CF}$.

\section{Atitudes ativas de envolvimento e participação}

Uma aprendizagem eficaz baseia-se em atitudes ativas de envolvimento e participação; é o «aprender fazendo» de John Dewey, tido como a melhor forma de adquirir conhecimento. ${ }^{11-12}$ Por isso, a CF promove momentos de prática, como workshops e treino de comunicações orais. No mesmo sentido, a aprendizagem na CF faz-se também pela análise e reflexão conjuntas por parte dos internos e dos orientadores sobre situações reais da prática clínica, sempre cultivando o princípio da confidencialidade. ${ }^{11-12}$ Acredita-se que a experiência prática promove a reflexão e que, desta, resultará a compreensão, por sua vez permitindo a aplicação prática de conceitos que criam uma nova experiência, retomando e potenciando o ciclo de aprendizagem ( $\mathrm{Fi}$ gura 1)..$^{13-14}$

\section{Corresponsabilização}

Sendo o internato de MGF um processo de aprendizagem de adultos, os resultados dos internos são essencialmente da sua responsabilidade; o que não diminui a responsabilidade dos orientadores em aperfeiçoar continuamente a qualidade do seu desempenho como mentores e como médicos de família, sendo o seu exemplo um contributo para a formação.

\section{Liberdade de interrogação crítica sobre atividades e práticas}

Todos se comprometem a defender e a promover a liberdade de consciência, a liberdade de perguntar, de refletir e de questionar o sentido, a fundamentação científica e a utilidade/impactos esperados de tudo o que se faz. 


\section{Responsabilidade e soli- dariedade}

Todos se comprometem a manter atitudes e comportamentos de responsabilidade e solidariedade no trabalho clínico e na formação no internato.

\section{Atividades desenvolvidas}

A cada semestre são eleitos dois coordenadores da $\mathrm{CF}$, um orientador e um interno, responsáveis por organizar as atividades e moderar as reuniões. As reuniões são agendadas semestralmente. Cada reunião é posteriormente documentada através da realização de uma ata, que detalha os vários temas tratados, a lista de presenças e as tarefas pendentes até à próxima reunião.

Durante os quatro anos de existência da CF, têm vindo a ser desenvolvidas as seguintes atividades:

\section{1) Reuniões periódicas dos orientadores - EIO, cujos} objetivos consistem em abordar aspetos organizativos gerais e locais do internato, bem como aspetos metodológicos e práticos da orientação dos internos. Estas reuniões têm uma frequência bimestral e a duração de uma hora, alternando ao longo do ano o dia da semana em que ocorrem, de modo a interferir o mínimo possível na agenda dos profissionais;

2) Reuniões periódicas dos internos - EII, cujos objetivos consistem em abordar aspetos organizativos gerais e locais do internato, bem como a partilha de experiências entre os internos. Estas reuniões têm uma frequência bimestral e a duração de uma hora, agendadas nas mesmas datas que as reuniões da EIO;

3) Reuniões periódicas conjuntas dos grupos de orientadores e internos-EIOI (equipa integrada de orientadores e internos), cujos objetivos consistem em abordar os aspetos organizativos gerais e locais do internato, bem como aspetos metodológicos e práticos do processo de aprendizagem e treino de competências. A estrutura das reuniões segue o modelo representado na Tabela 1, podendo ser alterada de acordo com as propostas existentes e necessidades formativas que possam surgir. Neste contexto podem surgir atividades como:

- Apresentação de artigos científicos (e.g., Social prescribing, da revista BRITISH MEDICAL JouRnAL);

- Apresentação de documentos de interesse para a CF (e.g., Educational training requirements for GP/FM specialist training, da European Academy of Teachers in General Practice EURACT);

- Exposição e feedback relativo a comunicações orais, posters, artigos, protocolos e outros trabalhos dos membros da CF (e.g., poster sobre prescrição de psicofármacos em Portugal);

- Revisões oportunas de um tema (e.g., Cefaleias, a propósito de um caso clínico);

- Apresentações de projetos finalizados ou em curso (e.g., Projeto Abrigar Saúde);

- Partilha de experiências de estágios e outras (e.g., estágios no estrangeiro); 
TABELA 1. Estrutura geral e exemplo de programa de reunião EIOI

\begin{tabular}{|c|c|c|}
\hline Estrutura geral do Programa & $\begin{array}{l}\text { Duração } \\
\text { (total } 2 \mathrm{~h} \text { ) }\end{array}$ & Exemplo de Programa \\
\hline Introdução, informações gerais & $\begin{array}{l}10 \text { a } 15 \\
\text { minutos }\end{array}$ & Receção, agenda e apresentação dos novos elementos da EIOI \\
\hline \multirow{4}{*}{$\begin{array}{l}\text { Apresentações diversas: } \\
\text { - Temas técnico-científicos ou } \\
\text { sobre formação } \\
\text { - Relatos de caso } \\
\text { - Relatos de prática } \\
\text { - Projetos de investigação } \\
\text { - Journal-club } \\
\text { - Workshops } \\
\text { - Debates } \\
\text { - Feedback de participação em } \\
\text { eventos formativos }\end{array}$} & \multirow{4}{*}{$\begin{array}{l}10 \text { a } 60 \\
\text { minutos } \\
\text { (cada item) }\end{array}$} & $\begin{array}{l}\text { Apresentação sobre o Guião de aprendizagem relacional e do novo programa } \\
\text { do Internato - o que há de novo. }\end{array}$ \\
\hline & & Apresentação do Projeto Abrigar Saúde \\
\hline & & $\begin{array}{l}\text { Journal-club: } \\
\text { - O caso do Dr. Bawa Garba } \\
\text { - Being a reflective practitioner }\end{array}$ \\
\hline & & Apresentação sobre Comunidades de prática e aprendizagem reflexiva \\
\hline $\begin{array}{l}\text { Atualização semestral do Jornal } \\
\text { da EIOI }\end{array}$ & $\begin{array}{l}15 \text { a } 20 \\
\text { minutos }\end{array}$ & Jornal da EIOI do Estoril \\
\hline Questões organizativas & $\begin{array}{l}10 \text { a } 25 \\
\text { minutos }\end{array}$ & $\begin{array}{l}\text { Planeamento das Jornadas da CF do Estoril e agendamento de reuniões para } \\
\text { o próximo semestre }\end{array}$ \\
\hline
\end{tabular}

- Dinamização de workshops de exame objetivo (e.g., workshop de exame osteoarticular);

Estas reuniões ocorrem com periodicidade bimestral, com a duração de duas horas, alternando ao longo do ano o dia da semana em que ocorre, à semelhança das reuniões descritas acima;

4) Experiências de estágio com outros orientadores e noutras unidades;

5) Elaboração do Jornal da Comunidade Formativa com periodicidade semestral, divulgado através de correio eletrónico, em que se pretende divulgar as atividades desenvolvidas nesta comunidade;

6) Participação em jornadas, reuniões formativas e outras atividades relevantes organizadas por qualquer das USF da CF;

7) Organização das I Jornadas da Comunidade Formativa, em julho de 2019. Estas primeiras jornadas foram subordinadas ao tema A Formação em Medicina Gerale Familiar no Século XXI. Procurou-se criar uma ferramenta de partilha e compilação de ideias entre participantes, disponível online e de livre acesso (usando a plataforma Padlet), que perdurasse além do momento das jornadas, podendo tornar-se um meio de extensão da atividade da CF a todos os interessados;

8) Implementação e manutenção de um fórum de discussão na internet que reúne todos os membros da CF como modo de manter o contacto entre reuniões e ferramenta muito útil de comunicação e partilha;

9) Incubadora de ideias - Durante os últimos anos têm sido apresentados alguns projetos na CF do Estoril que foram posteriormente implementados pelos internos e recém-especialistas. As EIOI funcionaram, assim, como «incubadora de ideias» para receber feedbacke angariar voluntários para os projetos. Dois exemplos são o projeto Abrigar Saúde, que visa o apoio médico a pessoas em condição de sem-abrigo no concelho de Cascais e a criação da Unidade de Cuidados Paliativos do ACeS Cascais.

\section{DISCUSSÃO}

O Internato Complementar de Clínica Geral, agora designado Formação Específica em MGF, é relativamente recente, tendo 38 anos de evolução. ${ }^{6}$ No seu começo, a formação de internos desta especialidade era feita nos hospitais e os orientadores de formação 
pertenciam à carreira hospitalar; o que significa que muitos dos atuais orientadores de formação não tiveram, eles próprios, acesso ao internato médico. Desta forma, a curta história do internato de MGF requisita a ativa participação daqueles que o integram, sendo as comunidades formativas «espaços» privilegiados para o fazer, contribuindo ativamente para a sua constante melhoria.

Em 2019, o colégio da especialidade prevê que todos os médicos internos e orientadores integrem uma Comunidade de Pratica Formativa, sendo desejavel que esta comunidade seja constituída, sempre que possivel, por elementos de diferentes unidades formativas, dentro da mesma instituicao ou em casos particulares de proximidade com outros servicos. A proximidade geografica e uma condição, mas nao e obrigação. A Comunidade de Prática Formativa deve ter por objetivos refletir sobre a formacao e o treino, partilhar a resolução de problemas, assim como encontrar formas comuns de os resolver, desenvolver guioes de procedimentos para situações específicas, partilhar recursos, aferir progressoes, metodos e avaliacões. Pretende-se que estas reuniões tenham um caráter mensal, incluindo na sua planificação a elaboração de uma ordem de trabalhos, o registo de assiduidades e respetiva ata. ${ }^{15}$ Reconhecendo o potencial das comunidades de prática, o novo programa de formação em Medicina Geral e Familiar (em vigor para os internos que iniciaram a formação específica a partir de 2019) prevê que quatro horas do horário semanal sejam alocadas a uma Sessão de Aprendizagem Relacional entre internos do mesmo ano, do mesmo ACeS, onde a partilha de conhecimento é o processo-chave. ${ }^{16-17}$

O percurso de cada interno é único, fruto de várias particularidades: a unidade que o acolhe, o orientador, a lista de utentes, os locais onde faz estágios, as oportunidades de aprendizagem que lhe surgem ou que procura. De igual modo, a partilha destas experiências únicas potencia as oportunidades de aprendizagem dos profissionais de MGF.

A integração de profissionais de outras especialidades, como a Saúde Pública, é uma mais-valia, pois permite alargar o âmbito dos temas em discussão e visões do sistema de saúde, o desenvolvimento de objetivos comuns às especialidades, a partilha de ideias, o aumento da aprendizagem e o desenvolvimento de frutíferas relações profissionais.
Esta metodologia organizativa só consegue ser eficaz e profícua se todos os seus intervenientes, de forma individual, cultivarem uma postura pró-ativa na organização e participação das sessões e dinâmicas calendarizadas. Esta postura é exigente e corresponde talvez ao maior desafio dentro de uma comunidade formativa.

\section{DESAFIOS PARA O FUTURO}

Um dos maiores desafios à continuidade da CF do Estoril tem sido a dificuldade na dinamização das reuniões e motivação contínua dos profissionais. Trata-se de atividades sem caráter obrigatório para orientadores ou internos e sem espaço próprio nos horários dos participantes, o que significa que a CF vive exclusivamente enquanto os profissionais o desejarem e mantiverem, correspondendo por este motivo a um desafio contínuo da equipa. Duas estratégias encontradas para lidar com a desmotivação e inércia foram a alteração semestral dos dinamizadores das reuniões da EIOI e sondagem online acerca dos temas de interesse para desenvolver nas próximas reuniões. Além disso, propõe-se que as reuniões aconteçam em dia fixo da semana; reduzir o tempo de duração para uma hora; salientar a importância de existir uma organização mais atempada das reuniões e de todos os elementos se comprometerem a participar mais na sugestão e apresentação de temas. Pretende-se ainda ter uma planificação anual dos temas a serem abordados nas várias reuniões e respetiva distribuição por todos os elementos da comunidade formativa. Foi sugerido, por último, que a organização das EIOI pudesse ficar a cargo de determinada USF, de forma rotativa.

A CF Estoril tem a ambição de cultivar-se num espírito crítico, curioso e potenciador de novas simbioses de trabalho e formação, trazendo ganhos educacionais e humanos para os seus elementos, bem como para a comunidade e outros organismos parceiros. Neste sentido, a CF continuará a trabalhar no sentido da criação de novos projetos colaborativos entre estas mesmas entidades; na promoção de novos projetos de investigação; e na utilização das novas tecnologias para a criação de grupos de discussão e partilha de conhecimento. Pretende-se também o reforço das experiências de âmbito prático com exercícios de treino específicos [e.g., segundo o modelo objective structured clinical examination (OSCE)] e promoção de workshops (e.g., 
sobre exame objetivo ou como preparação para a prova final de internato). Por último, é um desejo desta $\mathrm{CF}$ estabelecer as suas jornadas num evento anual de elevado valor científico, abordando temas fraturantes e inovadores para a MGF e internato.

\section{CONCLUSÃO}

Num discurso de graduação, David Foster Wallace conta a história de dois peixes jovens que nadam lado a lado num aquário. Conhecem um peixe mais velho que os cumprimenta dizendo: "Bom dia, jovens! Que tal a água?” Os peixes mais novos, confusos, continuam a nadar durante alguns segundos até que eventualmente um deles diz para o outro: "Mas que raio é a água?"18

O ritmo acelerado da prática clínica deixa aos profissionais pouco tempo para refletir acerca do que é feito, como é feito e para que é feito. A medicina e o seu ensino necessitam de espaços críticos, livres de acusações, onde profissionais com diferentes tipos de experiência e conhecimentos se desafiem mutuamente a melhorar as suas práticas. A partilha de uma experiência ou conhecimento exige o seu aprofundamento e pode desencadear novas ideias ou perceções sobre um assunto.

Acreditamos que as Comunidades Formativas têm o potencial de gerar esta autoconsciência e representam uma mais-valia para orientadores e internos, numa interação em que o todo é muito mais vasto do que a soma das partes. No caso da CF do Estoril, a proximidade gerada pelo trabalho no mesmo edifício permitiu aos membros das quatro unidades formar este projeto, num "misto de entusiasmo e familiaridade - focada na evolução, na geração de valor, aberta ao diálogo" ${ }^{5}$ e permeável ao que a rodeia - um pouco mais perto de vermos "a água que nos rodeia". ${ }^{18}$

\section{AGRADECIMENTOS}

Agradecemos a todos os elementos que fizeram e fazem parte da Comunidade Formativa do Estoril pelo seu contributo para o crescimento da mesma ao longo destes anos; à Dra. Cecília Shinn pelo desafio para a realização deste artigo; e, ainda, agradecemos os contributos de Francisco Carvalho, Teresa Ventura e Ricardina Barroso que muito valor acrescentaram a este trabalho.

\section{REFERÊNCIAS BIBLIOGRÁFICAS}

1. Comunidade Formativa do Estoril. Plano de atividades. São João do Estoril: CF Estoril; 2015.
2. Garvin DA. Learning in action: a guide to putting the learning organization to work. Boston: Harvard Business Review Press; 2000. ISBN 9781591391906

3. Wenger E, McDermott R, SnyderWM. Cultivating communities of practice. Boston: Harvard Business Review Press; 2002. ISBN 9781578513307

4. Von Krogh G, Ichijo K, Nonaka I. Enabling knowledge creation: how to unlock the mystery of tacit knowledge and release the power of innovation. Oxford: Oxford University Press; 2000. ISBN 9780195126167

5. Covita H. O papel das comunidades de práticas na prestação de cuidados de saúde primários [The role of communities of practice in the provision in primary health care]. Rev Port Clin Geral. 2006;22(1):819. Portuguese

6. Administração Regional de Saúde de Lisboa e Vale do Tejo. História do internato complementar de clínica geral [homepage]. Lisboa: ARSLVT; [s.d.] [cited 2019 Sep 16]. Available from: https://www.arslvt.min-saude.pt/pages/451

7. Coordenação do Internato Complementar de Clínica Geral da Zona Sul. Portfólio. Lisboa: CICCGZS; 2002.

8. Carvalho F, Ventura T, Barroso R. Perfil de competências do orientador de formação [Skills of the residency trainer]. Rev Port Clin Geral. 2004;20(1):147-52. Portuguese

9. Coordenação do Internato Médico de Medicina Geral e Familiar. Memorando sobre a formação em MGF e respetivo enquadramento. Lisboa: CIMGF; 2007.

10. Benetti IC, Vieira ML, Crepaldi MA, Schneider DR. Fundamentos da teoria bioecológica de Urie Bronfenbrenner [Fundamentals of the bioecological theory of Urie Bronfenbrenner]. Pensando Psicología. 2013;9(16):89-99. Portuguese

11. Reime MH, Johnsgaard T, Kvam Fl, Aarflot M, Engeberg JM, Breivik M, et al. Learning by viewing versus learning by doing: a comparative study of observer and participant experiences during an interprofessional simulation training. J Interprof Care. 2017;31(1):51-8.

12. Schmidt IA. John Dewey e a educação para uma sociedade democrática [John Dewey and the education for a democratic society]. Rev Contexto Educ. 2009;24(82):135-54. Portuguese

13. Colégio de Medicina Geral e Familiar. Perfil de competências do especialista em medicina geral e familiar [homepage]. Lisboa: Ordem dos Médicos; 2019. Available from: https://ordemdosmedicos.pt/perfil-decompetencias-do-especialista-em-medicina-geral-e-familiar/

14. Sims RR. Kolb's experiential learning theory: a framework for assessing person-job interaction. Acad Manage Rev. 1983;8(3):501-8.

15. Colégio de Medicina Geral e Familiar. Requisitos para obtenção de idoneidade formativa [Internet]. Lisboa: Ordem dos Médicos; 2019. Available from: https://ordemdosmedicos.pt/wp-content/uploads/2019/ 05/CIF-MGF-2019.08.01.pdf

16. Portaria n. ${ }^{\circ}$ 125/2019, de 30 de abril. Diário da República. I Série;(83).

17. Coordenação do Internato Médico de Medicina Geral e Familiar. Manual de formação: versão 2019.b [2019-04-30] [Internet]. Lisboa: CIMGF; 2019. Available from: https://docplayer.com.br/151746962Manual-de-formacao-versao-2019-b.html

18. Krajeski J. This is water. The New Yorker [Internet]; 2008 September 19. Available from: https://www.newyorker.com/books/page-turner/thisis-water 


\section{CONFLITO DE INTERESSES}

Os autores declaram não ter quaisquer conflitos de interesse.

ENDEREÇO PARA CORRESPONDÊNCIA

Vanessa Sá
E-mail: svanessa.sa@gmail.com

https://orcid.org/0000-0003-3877-790X

Recebido em 14-11-2019

Aceite para publicação em 20-05-2020

\section{ABSTRACT \\ ESTORIL TRAINING COMMUNITY: A COMMUNITY OF PRACTICE FOCUSED ON THE MEDICAL RESIDENCY AND LOOKING INTO THE FUTURE}

Introduction: A community of practice is a group of people coming together around the same topic or interest, working together to improve what they do through regular interactions. The Estoril Training Community brings together a group of residents and attending physicians in Family Medicine and Public Health in the region of Estoril (Lisbon Metropolitan Area, Portugal) with the aim of broadening their range of experiences, reflecting the principles of a community of practice. This article reports the experience of the Estoril Training Community and its main objective is to make their practices known, to encourage the sharing of experiences between groups, and inspire the formation of new communities.

Description: The Estoril Training Community was born in 2015 and was composed by residents and attending physicians in family medicine and public health from four of the functional units of the São João do Estoril's healthcare center, in Cascais. Since the establishment of the Community, there have been various activities such as periodic meetings, preparation of the Training Community newspaper, organization of the 1st Conference of the Estoril Training Community, among others.

Conclusion: The Training Community is an asset for mentors and interns, in an interaction where the whole is larger than the sum of the parts. In the case of the Estoril Training Community, the proximity originated by working in the same building allowed its members to create this project in a collaborative and mutual help atmosphere - a productive community, open to dialogue, permeable to its surroundings and with the desire and ability to evolve through the input of each new member and the interactions that are created from there.

Keywords: Medical education; Training community; Internship and residency. 